\title{
Arteriovenous Fistula Toxicity
}

\author{
Richard Amerling ${ }^{a}$ Claudio Ronco $^{b}$ Martin Kuhlmann ${ }^{c}$ James F. Winchester ${ }^{d}$ \\ a Department of Internal Medicine - Nephrology, Vivantes Klinikum im Friedrichshain, Berlin, Germany; \\ ${ }^{b}$ Division of Nephrology and Transplantation, Hospital St. Bortolo, Vicenza, Italy; ' Department of Nephrology,

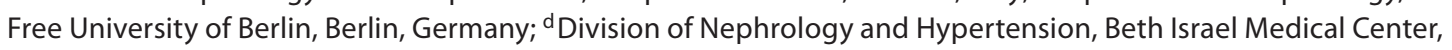 \\ New York, N.Y., USA
}

\section{Key Words}

Arteriovenous fistula - Hemodialysis - Vascular access •

Cardiovascular mortality

\begin{abstract}
The arteriovenous fistula (AVF) has been a mainstay of hemodialysis treatments and the preferred access route since its inception in the 1960s, due to its longevity and resistance to infection. However, the AVF is not benign. There is significant primary failure, as well as cardiac, vascular, and other, less well recognized, complications. Together, they represent toxicity, to which considerable morbidity and mortality can be attached. Official policy, based on guidelines where AVF toxicity is given short shrift, drives an increase in use of these devices, and may have undesired consequences.
\end{abstract}

Copyright $\odot 2011$ S. Karger AG, Basel

\section{Introduction}

The Brescia-Cimino arteriovenous fistula (AVF) is accurately credited with making chronic hemodialysis (HD) a viable long-term treatment [1]. The use of endogenous vessels granted longevity and resistance to infection. Over recent years, enthusiasm for this procedure has morphed into an almost irrational embrace. Obvious problems associated with the creation of large leftto-right shunts are swept under the rug in a publicity campaign designed only to extol the virtues, and to demonize alternatives. The original Brescia-Cimino procedure was a radial artery to cephalic vein anastomosis. While still preferred, it is slowly giving way to the much larger, and potentially more dangerous, brachiocephalic (BC) and brachiobasilic shunts [2]. Use of the AVF became mainstream without any prospective trials and with minimal consideration of long-term negative effects.

Consider the following questions:

- Why don't HD patients, even those who begin dialysis young, survive beyond twenty or so years?

- Why is cardiovascular (CV) mortality so much higher in the hemodialysis population, as compared with the age-matched general population? [3]

- Why are traditional risk factors for CV disease not associated with mortality in HD patients (in fact, they have an inverse relationship to mortality)? A corollary question is: why do $\mathrm{CV}$ interventional trials fail to show benefit in HD patients? [4]

- Why is survival in preemptive transplant patients higher than in those transplanted after initiation of HD? [5]

\section{KARGER \\ Fax +41613061234 \\ E-Mail karger@karger.ch}

www.karger.com
(C) 2011 S. Karger AG, Basel

0253-5068/11/0313-0113\$38.00/0

Accessible online at:

www.karger.com/bpu
Richard Amerling, MD

Division of Nephrology and Hypertension

Beth Israel Medical Center

350 East 17th Street, New York, NY 10003 (USA)

E-Mail ramerlin@chpnet.org 


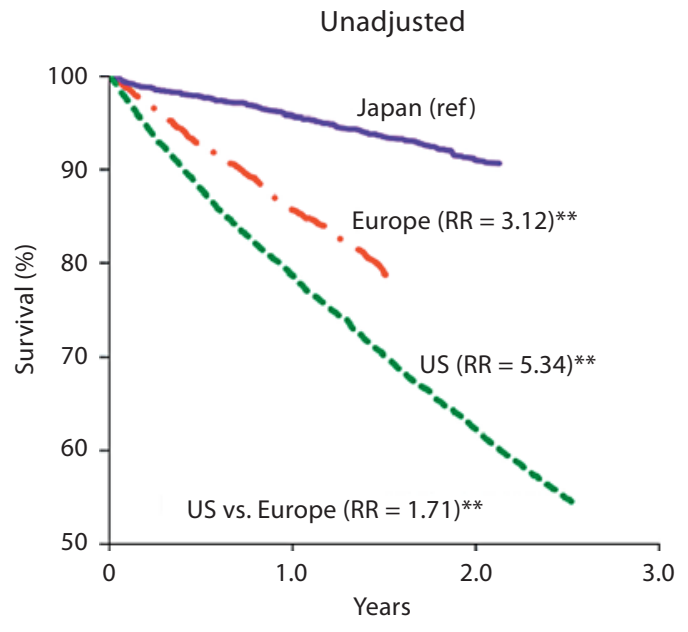

DOPPS

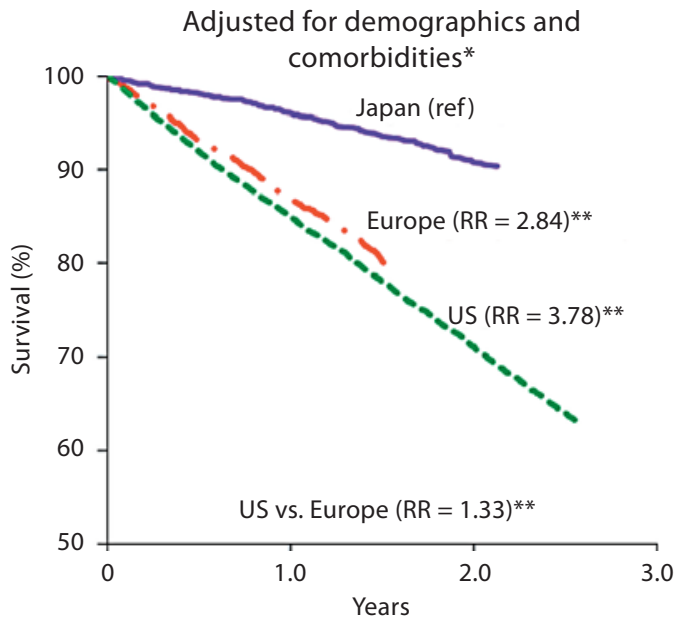

Robinson, BM and Port, FK. CJASN 4: S12-S17, 2009
Fig. 1. Survival on HD in Japan is much better than in the US and Europe, even adjusted for demographics and comorbidities (http://www.dopps.org/show_Citation.aspx?id=422). Data from DOPPS I (1997 through 2001). * Cox proportional hazards model adjusted for age, male gender, black race, coronary artery disease, congestive heart failure, other cardiac disease, left ventricular hy- pertrophy, cardiomegaly by X-ray, hypertension, cardiovascular disease, peripheral vascular disease, diabetes, lung disease, dyspnea, smoking, cancer, HIV/AIDS, gastrointestinal bleed, peptic ulcer disease, hepatitis $\mathrm{B}$, hepatitis $\mathrm{C}$, neurologic disorder, psychiatric disease, recurrent cellulitis or gangrene, and vision problems. ${ }^{* *} \mathrm{p}<0.001$.

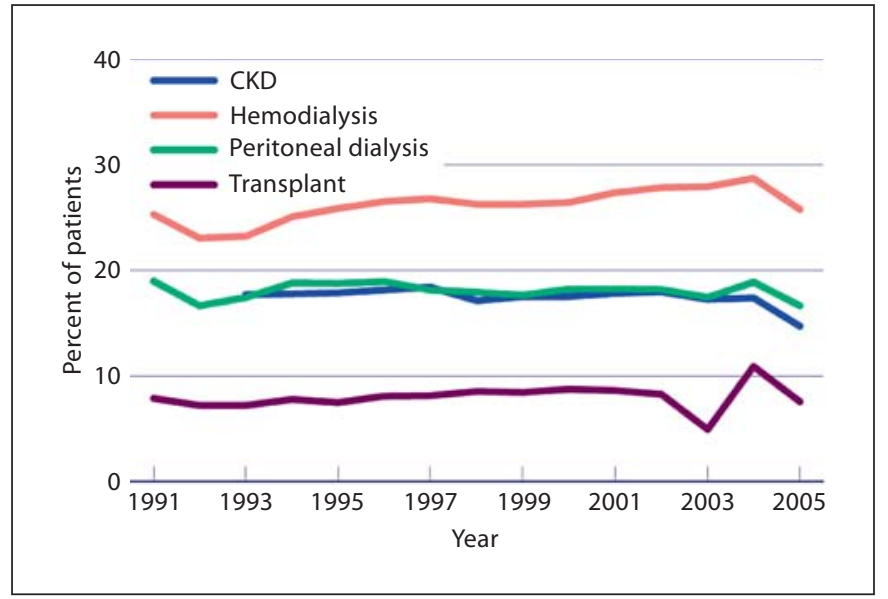

Fig. 2. Incidence of $\mathrm{CHF}$ in prevalent patients (USRDS 2007 ADR). CKD signifies point prevalent general Medicare CKD patients (from the 5\% sample), age 66 and older; adjusted for age, gender, race, and diabetic status. ESRD signifies point prevalent ESRD patients; adjusted for age, gender, race, and dialysis vintage.

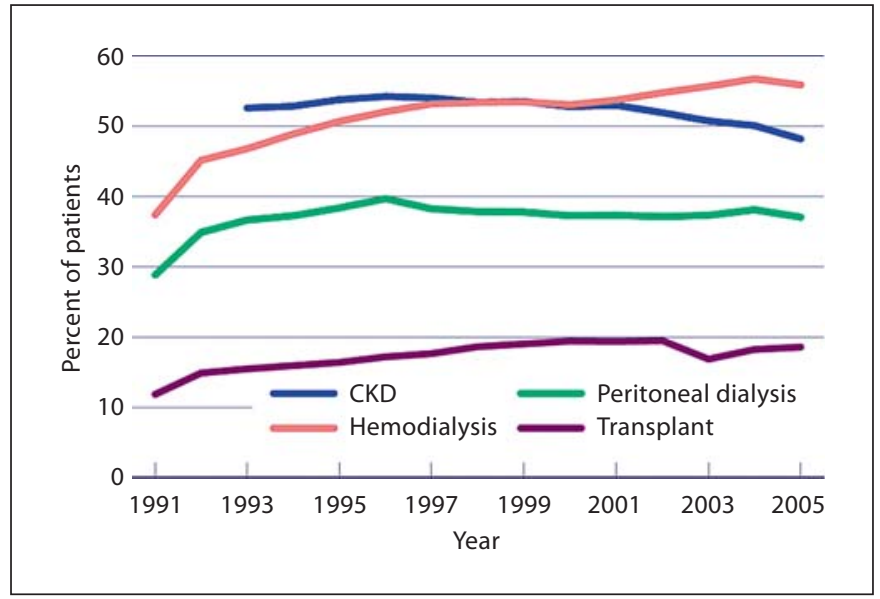

Fig. 3. Prevalence of CHF in prevalent patients (USRDS 2007 ADR). CKD signifies point prevalent general Medicare CKD patients (from the 5\% sample), age 66 and older; adjusted for age, gender, race, and diabetic status. ESRD signifies point prevalent ESRD patients; adjusted for age, gender, race, and dialysis vintage. 


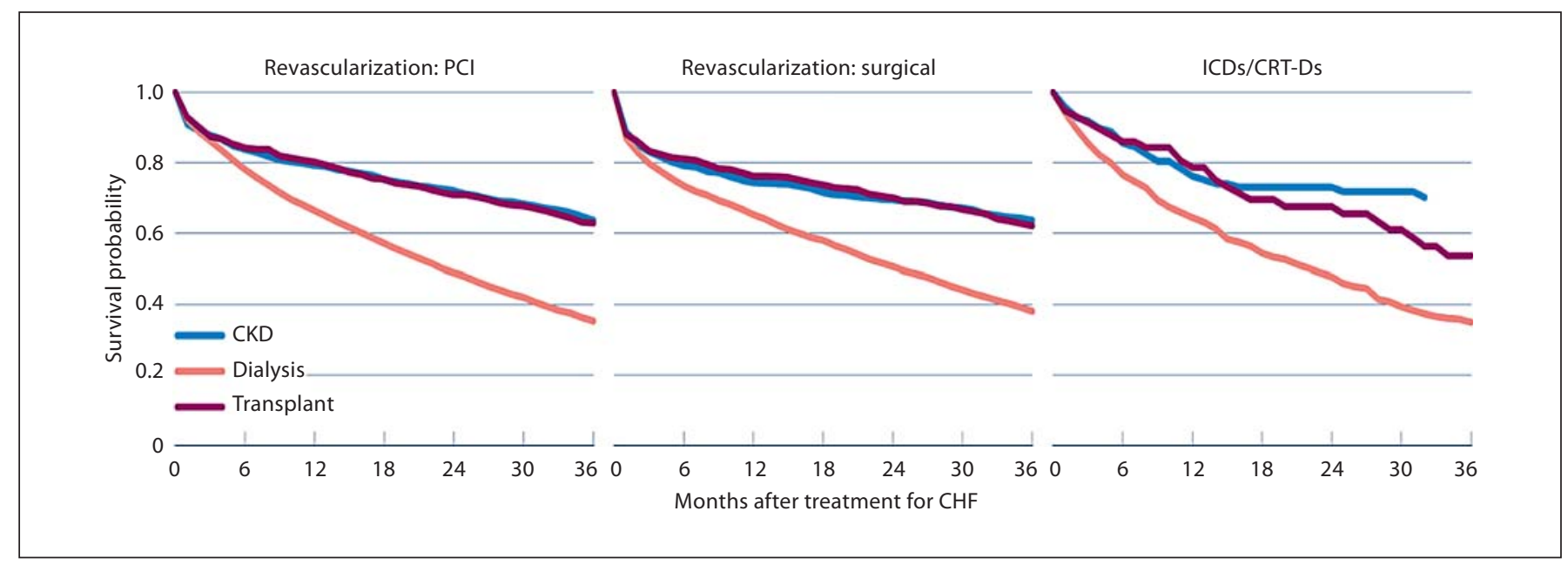

Fig. 4. Survival after treatment for CHF, by type of treatment (USRDS 2007 ADR). Incident CHF patients, 1996-2003, receiving treatment within one year after CHF diagnosis, unadjusted. CKD patients: age 66 and older; ESRD patients: age 20 and older. $\mathrm{PCI}=$ Percutaneous coronary intervention; ICDs/CRT-Ds = implantable cardiac defibrillator/cardiac resynchronization therapy-defibrillator.

- Why do Japanese HD patients have so much lower mortality than US HD patients? (fig. 1) [6]

- Why do peritoneal dialysis (PD) patients have lower incidence and prevalence of congestive heart failure (CHF) than HD patients? (fig. 2, 3)

- Why is survival after CV procedures such as revascularization so dismal in HD patients? (fig. 4)

- Why hasn't the increased prevalence of AVF been associated with an improved first year mortality? (fig. 5, 6)

- Would a surgeon knowingly create an AVF in an otherwise healthy patient? Would a surgeon refuse to ligate a traumatic AVF?

The answers all relate to the AVF. Even relatively young and healthy patients will eventually develop CV complications, mostly CHF, due to the prolonged presence of an AVF. CV mortality that is orders of magnitude greater in young HD patients compared with the general population cannot only be due to traditional CV risk factors. Studies in HD patients of modification of CV risk factors, including calcium loading, fail to show significant benefit because the risk associated with an AVF creates 'noise' that drowns out any effect of treatment. Preemptive transplant patients usually do not have an AVF, while those transplanted after initiation of HD often do. The Japanese perform HD with lower blood flow rates and typically create relatively small radiocephalic (RC) AVF, which presumably are a lesser cardiac burden. PD patients, for the most part, do not have an AVF. HD patients do poorly after revascularization because an AVF in addition to coronary artery disease (CAD), or established CHF, is a disaster. Mortality has not improved overall with greater use of AVFs because they are likely being placed in some patients who should not receive them based on clinical status. The last two questions are obviously rhetorical.

This paper is an attempt to restore critical thinking to this crucial area of hemodialysis treatment. We use the term AVF toxicity to describe a constellation of negative effects directly related to the presence of an AVF. Some effects, such as high output heart failure, are obvious, though underrecognized. Others, including underdialysis, malnutrition, and global steal syndrome, are less so, and are described here for the first time. We conclude with recommendations for vascular access policy that take into account and seek to minimize AVF toxicity.

\section{Cardiac Toxicity}

The hemodynamic effects of a left-to-right shunt have been well described (table 1) [7-10]. With creation of an AVF, there is an immediate drop in peripheral vascular resistance, which leads to an immediate, compensatory increase in cardiac output (CO). This is accomplished by increasing pulse rate, stroke volume, and contractility, all mediated by activation of the sympathetic nervous system. 


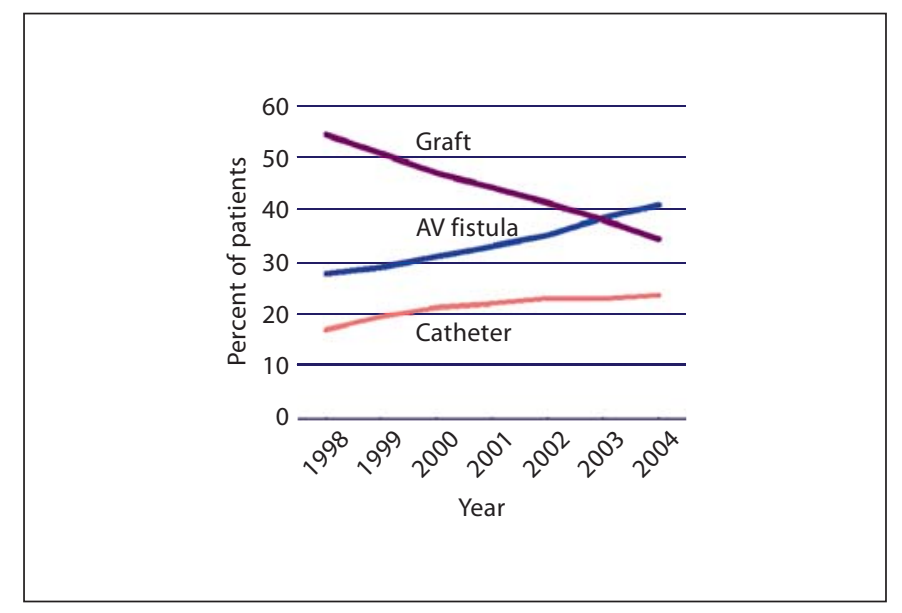

Fig. 5. Vascular access use by type (USRDS 2007 ADR). December 31 point prevalent hemodialysis patients; ESRD CPM data. Access represents the current access as of the latest data collection for the year. Includes only patients for whom the access is known.

Table 1. Hemodynamic effects of AVF

- Increased CO

- Decreased peripheral resistance

- Increased sympathetic activity with increased contractility, heart rate and stroke volume

- Increased blood volume with increased ANP and BNP, LVEDD

- Increased pulmonary flow and pressure

$\mathrm{ANP}=$ Atrial natriuretic peptide; $\mathrm{BNP}=$ brain natriuretic peptide; LVEDD = left ventricular end diastolic dimension.

Atrial and brain natriuretic peptide levels increase. There is expansion of the blood volume with increased left ventricular end diastolic dimension. Pulmonary flow (and in dialysis patients, pulmonary pressure) increases [11].

Over time, there is cardiac hypertrophy and four-chamber enlargement. Diastolic dysfunction and CHF may occur [12]. Classic high output failure has been described in numerous case reports $[13,14]$, but is very likely underrecognized [15]. It should be considered in any dialysis patient presenting with signs or symptoms of CHF, but without obvious volume overload. Repeated hospital admissions, with variable response to HD are further clues. These patients should be investigated with right heart catheterization and measurement of $\mathrm{CO}$ before and after AV shunt

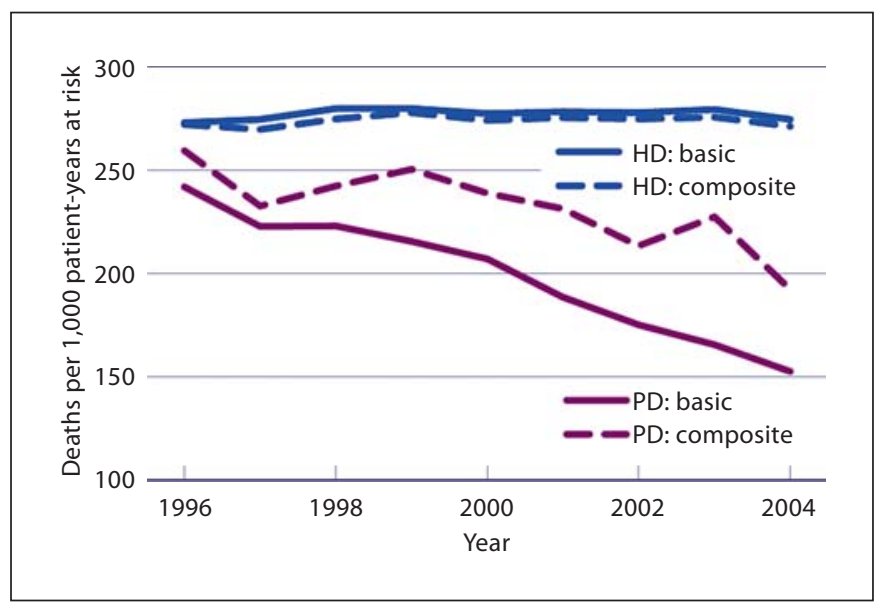

Fig. 6. Dialysis first-year mortality rate, with basic versus composite adjustments (USRDS 2007 ADR). Incident dialysis patients. Basic adjustment: age, gender, race, and primary diagnosis. Composite adjustment: age, gender, race, primary diagnosis, comorbidities, BMI, hemoglobin, and eGFR. Comorbidities and laboratory information from the Medical Evidence form. Incident dialysis patients, 2004, used as reference cohort.

compression. It must be understood that measured $\mathrm{CO}$ may be normal in these patients [16]. We refer to this as 'pseudonormalization' of CO. It is the fraction of CO going to the AVF that is important [17]. If a patient with an AVF has symptomatic CHF, chamber dilatation, a reduced ejection fraction (EF), and a CO measured at $5 \mathrm{l} / \mathrm{min}$, we cannot be misled by the latter number. We refer to the 'effective cardiac output' (eCO) as the difference between the total CO and the AVF flow. This is the CO that is available for tissue perfusion. Since measurement of $\mathrm{CO}$ via thermodilution is not exactly precise, these numbers need to be interpreted using clinical judgment and experience. It is also unlikely that the $\mathrm{CO}$ will abruptly return to baseline, even after 5 or $10 \mathrm{~min}$ of shunt occlusion, due to ongoing activation of the sympathetic nervous system.

$$
\mathrm{eCO}=\text { Total CO - Qavf }
$$

Pulmonary hypertension has been detected in $40-50 \%$ of HD patients [18]. This morbid complication develops shortly after AVF creation, is related to the magnitude of the AV shunt, and is reversed by shunt ligation [19]. It is suggested that HD patients have inadequate pulmonary vasodilatation in response to the increased flows caused by the AVF, possibly due to suboptimal production of nitric oxide (NO), or the presence of inhibitors of NO, such as asymmetric dimethyl arginine [20]. 
The subendocardial viability index (SEVI) is a pressure-time integral ratio, where the numerator is the area between LV and Ao diastolic pressures and the denominator is area under LV systolic pressure. The numerator reflects coronary perfusion and the denominator the work of the heart. Decreases in the SEVI lead towards subendocardial ischemia, and presumably, fibrosis. It is clear that AVF decreases the SEVI, and the higher the fistula flow, the more this occurs [21]. Thus, AVFs set the stage for ischemic cardiomyopathy, which is the end stage of AVF-related cardiotoxicity.

AVF-related cardiac toxicity thus can have myriad presentations (table 2). We described several typical presentations in a case series [11]. There can be clear-cut high output failure with $\mathrm{CO}>10 \mathrm{l} / \mathrm{min}$. The insidious development of CHF over years in a patient with an AVF should cause concern, particularly if CO appears 'normal', and there is no other obvious explanation for CHF, such as severe CAD. Patients may develop acute decompensation of CHF shortly after creation, or repair, of an AVF or arteriovenous graft (AVG). Pulmonary hypertension is prima facie evidence of AVF toxicity, and the AVF should be considered a reversible aggravating factor in $\mathrm{CHF}$ or CAD in all patients.

\section{Steal Syndromes}

Everyone is familiar with the classical steal syndrome involving the extremity distal to an AVF, where circulation and perfusion is 'stolen' by the shunt (fig. 7). Less well known is the coronary steal syndrome, where a left-sided AVF 'steals' flow from a left internal mammary artery coronary bypass graft. This can lead to myocardial ischemia, which is sometimes reported to increase during hemodialysis [22].

We describe for the first time the 'global steal syndrome'. This occurs in compromised vascular beds removed from the actual site of the AVF, and is due to fistula-induced loss of eCO. Thus, lower extremity, or gut ischemia can be partly due to an upper arm AVF that robs a significant percentage of the $\mathrm{CO}$. We recently saw a patient with a BC AVF (and ipsilateral hand ischemia) who presented with bowel ischemia, evidenced by pneumatosis coli on CT scanning. After a banding procedure (Miller procedure), the intestinal ischemia resolved clinically and radiographically (fig. 8, 9). In AVF patients with refractory ischemia, fistula reduction or ligation should be considered as salvage therapy if other measures fail.

Arteriovenous Fistula Toxicity

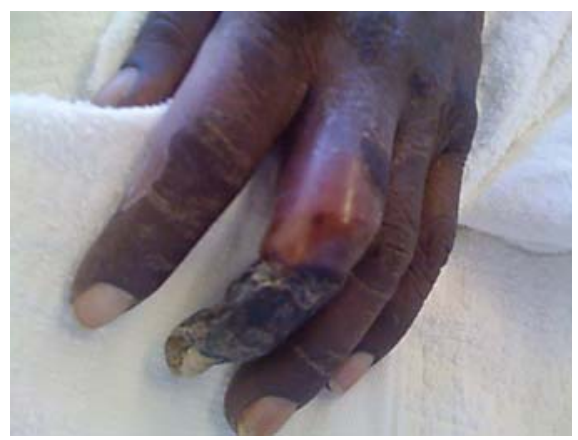

Fig. 7. Digital ischemic gangrene in HD patient with ipsilateral BC AVG.

Table 2. Cardiac toxicity of AVF

- LV dilatation; four-chamber enlargement

- Diastolic dysfunction

- CHF (high output)

- Decreased SEVI

- Pulmonary hypertension

- Cardiomyopathy

\section{Cardiopulmonary Recirculation, Underdialysis and Wasting}

Cardiopulmonary recirculation (CPR) was first described by Schneditz et al. [23] using thermal recirculation data generated by us. It is unique to fistulas and grafts, and decreases the efficiency of hemodialysis. Freshly dialyzed blood returns to the fistula and makes a rapid transit through the right heart and pulmonary circulation. It then is pumped into the systemic circulation by the left ventricle, but a portion of this dialyzed blood re-enters the AVF, largely undiluted. The magnitude of this effect is proportional to the percent of $\mathrm{CO}$ that goes to the AVF. Since this percentage increases with higher flow, upper arm fistulas, CPR must also increase with these shunts. Van der Mark et al. [24] measured AVF flow and $\mathrm{CO}$ using the Transonics system in RC and BC AVFs and AVGs (table 3). Average flow in the RC shunts was $1 \mathrm{l} / \mathrm{min}$, which represented $17 \%$ of CO. In the BC shunts, flow averaged $1.8 \mathrm{l} / \mathrm{min}$, which was $27 \%$ of CO. CPR increased from 17 to $28 \%$, respectively. CPR very likely contributes to underdialysis, malnutrition and wasting. There is no need for AVF flows over $600 \mathrm{ml} / \mathrm{min}$. Since higher flows actually reduce dialysis efficiency through CPR, it would make sense to surgically reduce upper arm shunts, where flows average $1,700 \mathrm{ml} / \mathrm{min}$.

Blood Purif 2011;31:113-120 


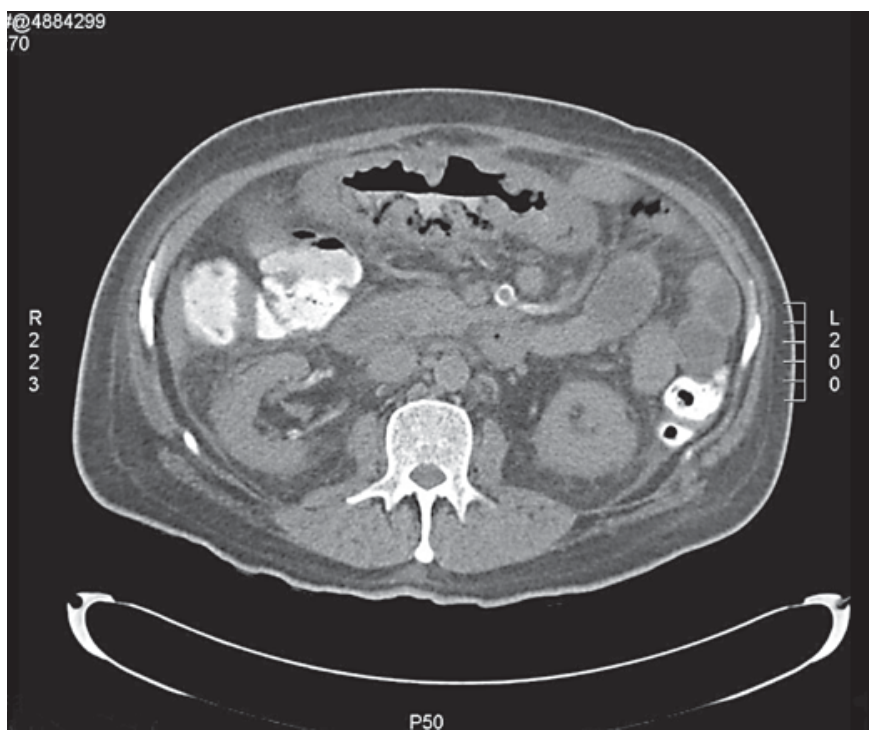

Fig. 8. Pneumatosis coli in HD patient with abdominal pain, prebanding of AVF.

Table 3. Shunt flows and resistances of hemodialysis patients with various shunt types [24]

\begin{tabular}{lccc}
\hline & $\begin{array}{l}\text { AVF } \\
\text { upper arm }\end{array}$ & $\begin{array}{l}\text { AVF } \\
\text { forearm }\end{array}$ & $\begin{array}{l}\text { PTFE } \\
\text { graft }\end{array}$ \\
\hline Patients & 32 & 39 & 15 \\
Qa, 1 & $1.8 \pm 0.2$ & $1.0 \pm 0.1^{* * *}$ & $0.7 \pm 0.1^{* * *}$ \\
CO, 1 & $6.6 \pm 0.3$ & $5.3 \pm 0.3^{* *}$ & $4.5 \pm 0.4^{* * *}$ \\
CPR ratio & $0.28 \pm 0.02$ & $0.17 \pm 0.01^{* * *}$ & $0.16 \pm 0.01^{* * *}$ \\
Adjusted Qa ${ }^{\text {a }}, 1$ & $1.5 \pm 0.1$ & $1.0 \pm 0.1^{* * *}$ & $0.9 \pm 0.2^{* * *}$ \\
MAP, mm Hg & $95 \pm 3$ & $91 \pm 3$ & $91 \pm 3$ \\
TPVR, mm Hg l I $^{\text {U1 }}$ & $16 \pm 1$ & $19 \pm 1$ & $22 \pm 2^{*}$ \\
AR, mm Hg l & $71 \pm 9$ & $142 \pm 16^{* * *}$ & $160 \pm 29^{* * *}$ \\
SVR, mm Hg l & $23 \pm 2$ & $23 \pm 1$ & $26 \pm 2$ \\
\hline
\end{tabular}

${ }^{* * *} \mathrm{p}<0.001 ;{ }^{* *} \mathrm{p}<0.01 ;{ }^{*} \mathrm{p}<0.02$ versus AVF upper arm group. $\mathrm{Qa}=$ Access flow; $\mathrm{MAP}=$ mean arterial pressure; $\mathrm{TPVR}=$ total peripheral vascular resistance; $\mathrm{AR}=$ access resistance; SVR = systemic vascular resistance.

${ }^{\mathrm{a}} \mathrm{Q}$ a adjusted by analysis of covariance for variations related to variations in $\mathrm{CO}$.

Adding to concerns about inadequate solute clearance is that by increasing $\mathrm{CO}$ and work of the heart, the AVF raises resting energy expenditure (REE). $\mathrm{CO}$ is a principle determinant of REE; thus, all AVFs increase REE in direct proportion to their effect on CO. Dialysis patients are on the borderline of protein-calorie malnutrition, and

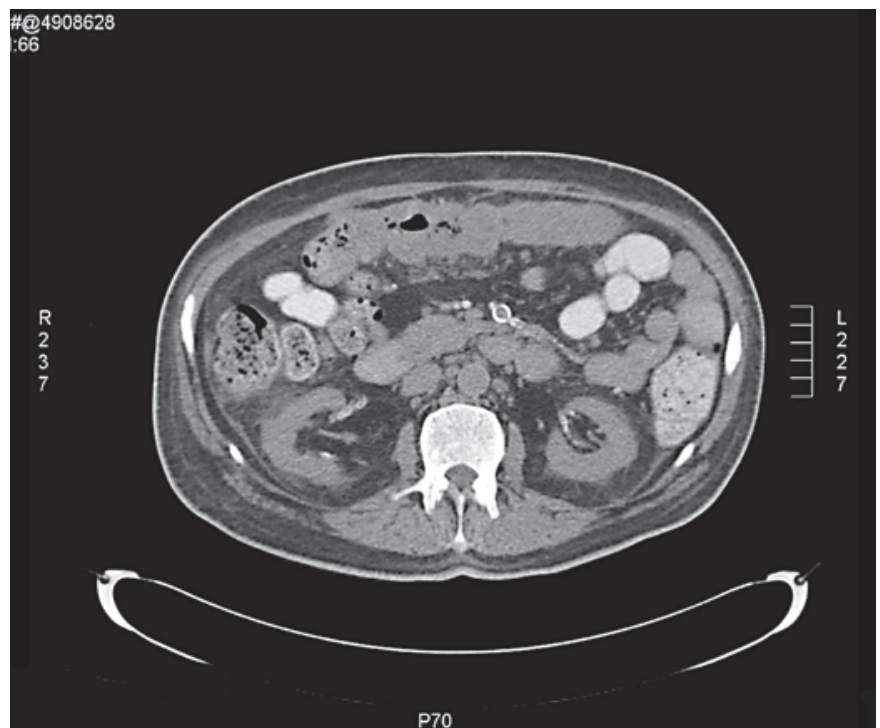

Fig. 9. Post-banding of AVF: clinically improved and pneumatosis absent.

this added energy requirement may contribute to longterm negative energy balance and wasting. HD patients with AVF who exhibit 'failure to thrive' should be considered for fistula ligation.

\section{Hypertension and Intradialytic Hypotension}

The hemodynamic response to an AVF as detailed above may cause sustained increases in sympathetic tone. The maintenance of a higher $\mathrm{CO}$ also requires an increased blood volume. These may lead to worsened, and or refractory, hypertension in certain patients. Some patients may also become pre-load dependent and have severe intradialytic hypotension with modest volume removal.

\section{Central Vein Stenosis}

MacRae et al. [25] suggest central vein stenoses develop in part as a result of vascular remodeling in response to high-flow AVFs. They base this on the observation of central vein stenoses in patients who never had central catheters, and that the majority of such stenoses occur at sites never cannulated. We believe this is an accurate assessment. Perhaps central vein stenoses are an attempt by the venous system to defend itself from ab- 
normally high pressure and flow? Perhaps we should allow these to develop (to a point) rather than obsessively dilating and stenting them?

\section{Natural History of the AVF}

Snapshot epidemiologic data and retrospective studies do not convey an adequate picture of the AVF toxicity [26]. There have been no randomized, long-term, controlled trials comparing AVF with AVG, or catheters. Obvious selection bias makes it impossible to draw conclusions from observational data on access choice and survival [27].

Observation of many patients over a long time leads to an understanding of the potentially sinister natural history of the AV shunt.

Immediately after creation of an AVF, the CO and work of the heart increase, while the SEVI deteriorates. Over a variable period of time, depending on baseline cardiac status, fistula flow, and other factors, the cardiac chambers dilate, and pulmonary hypertension develops. All roads lead to cardiomyopathy. The elevated CO declines toward 'pseudonormalization'. The AVF tends to dilate over time, with increased flows. It comes to steal a greater percentage of the reduced CO, and CPR increases, with impaired dialysis adequacy. Further reduction in the eCO leads to global hypoperfusion ('global steal syndrome'), failure to thrive, and death.

\section{Reversal of AVF Toxicity}

Several published studies confirm the reversibility of at least the cardiac manifestations of AVF toxicity [28, 29]. We have observed dramatic clinical and echocardiographic resolution of severe, symptomatic $\mathrm{CHF}$ in 3 patients following AVF closure and permcath placement. In a recent paper [30], $25 \mathrm{HD}$ patients with malfunctioning AVF who underwent fistula closure and permcath insertion were compared with 36 matched controls with functioning AVF. The closure group had significant decreases in LV mass, LV mass index, LV diastolic diameter, septal thickness, diastolic posterior wall thickness, and significant increases in EF, compared with no significant changes in the control group. The group that underwent ligation had relatively low fistula flow rates, due to chronic malfunction, suggesting the potential for more dramatic improvements in patients with high-flow fistulas.

\section{Discussion and Conclusions}

The AVF is a nonphysiologic anomaly and should be considered a 'lesser evil,' as opposed to something inherently desirable [31]. AVF toxicity is directly proportional to access flow rate, and inversely proportional to cardiac status. Fistula toxic effects are, for the most part, unrecognized and fly below radar. The AVF undoubtedly contributes to excess CV mortality in HD patients and shortens life spans.

Pressure to increase fistula use may worsen outcomes as clinically inappropriate patients receive them [32]. Cardiac evaluation with, at a minimum, echocardiography should precede AVF surgery. Patients with EF below $40 \%$ and significant pulmonary hypertension should not get an AVF.

High AVF flow is detrimental in many ways. A reasonable goal should be $400-800 \mathrm{ml} / \mathrm{min}$. We should learn from the Japanese. Brachial artery fistulas should be placed rarely, if at all. Every attempt should be made to moderate flow if they must be used. Cardiac status and fistula flows should be monitored; banding or ligation of AVF should be performed if the former is deteriorating. Outflow stenoses in high-flow AVF should be tolerated unless flows drop to $<500 \mathrm{ml} / \mathrm{min}$.

Catheters have obvious problems, but have been demonized mostly by associative data. There will be a need for catheters that cannot be reduced below a certain minimum. We can and must do a better job to reduce catheter complications.

PD should be the first modality in most, to spare them an AVF. We do not recommend placing a fistula electively in PD patients. The timing of placement of AVFs in predialysis patients needs to be considered carefully. Three months before anticipated start of HD should be more than adequate. It is unreasonable to give patients the vascular burden of an AVF a year or more before onset of HD. As a corollary, AV shunts should be ablated following successful transplantation, certainly after a year, and in some cases sooner.

In patients with refractory heart failure, unstable angina, global or peripheral steal syndrome, and failure to thrive, ligation of AVF is beneficial and indicated. 


\section{References}

-1 Brescia MJ, Cimino JE, Appel K: Chronic hemodialysis using venipuncture and a surgically created arteriovenous fistula. N Engl J Med 1966;275:1089-1092.

-2 Fitzgerald JT, Schanzer A, Chin AI, McVicar JP, Perez RV, Troppmann C: Outcomes of upper arm arteriovenous fistulas for maintenance hemodialysis access. Arch Surg 2004; 139:201-208.

$\checkmark 3$ Foley RN, Parfrey PS: Cardiovascular disease and mortality in ESRD. J Nephrol 1998; 11:239-245

$\checkmark 4$ Foley RN: Cardiac disease in chronic uremia: can it explain the reverse epidemiology of hypertension and survival in dialysis patients? Semin Dial 2004;17:275-278.

5 Papalois VE, Moss A, Gillingham KJ, Sutherland DE, Matas AJ, Humar A: Pre-emptive transplants for patients with renal failure: an argument against waiting until dialysis. Transplantation 2000;70:625-631.

6 Robinson BM, Port FK: International hemodialysis patient outcomes comparisons revisited: the role of practice patterns and other factors. Clin J Am Soc Nephrol 2009; 4(suppl 1):S12-S17.

7 Guyton AC, Sagawa K: Compensations of cardiac output and other circulatory functions in areflex dogs with large AV fistulas. Am J Physiol 1961;20:1157-1163.

$\checkmark 8$ Johnson G, Blythe WB: Hemodynamic effects of arteriovenous shunts used for hemodialysis. Ann Surg 1970;171:715-723.

$\checkmark 9$ Abassi ZA, Winaver J, Hoffman A: Large A-V fistula: pathophysiological consequences and therapeutic perspectives. Curr Vasc Pharmacol 2003;1:347-354.

-10 MacRae JM, Pandeya A, Jumen DP, Krivitski N, Lindsay RM: Arteriovenous fistula-associated high-output cardiac failure: a review of mechanisms. Am J Kidney Dis 2004; 43:e17-e22.

-11 Nakhoul F, Yigla M, Gilman R, Reisner SA, Abassi Z: The pathogenesis of pulmonary hypertension in haemodialysis patients via arterio-venous access. Nephrol Dial Transplant 2005;20:1686-1692.

- 12 von Bibra H, Castro L, Autenrieth G, McLeod A, Gurland HJ: The effects of arteriovenous shunts on cardiac function in renal dialysis patients - an echocardiographic evaluation. Clin Nephrol 1978;9:205-209.
13 Jin H, Afonso L, Singh A, Migdal S, Spears JR: Case report: recurrent heart failure with preserved ejection fraction but markedly elevated BNP in a 51-year-old female on hemodialysis with oversized AV fistula. Int J Cardiol 2006;110:429-430.

14 Young PR Jr, Rohr MS, Marterre WF Jr: High-output cardiac failure secondary to a brachiocephalic arteriovenous hemodialysis fistula: two cases. Am Surg 1998;64:239-241.

15 Engelberts I, Tordoir JH, Boon ES, Schreij G: High-output cardiac failure due to excessive shunting in a hemodialysis access fistula: an easily overlooked diagnosis. Am J Nephrol 1995;15:323-326.

16 Amerling R, Malostovker I, Dubrow A Rosero H, Haveson S: High output heart failure in patients with upper arm A-V fistulae: diagnosis and treatment. Blood Purif 2004; 22:389.

17 MacRae J, Pandey S, Hume D, Krivitski N, Lindsay R: A case report and review of arteriovenous fistula and high output cardiac failure. Am J Kidney Dis 2004;43:E21-E25.

-18 Yigla M, Nakhoul F, Sabag A, Tov N, Gorevich B, Abassi Z, Reisner SA: Pulmonary hypertension in patients with end-stage renal disease. Chest 2003;123:1577-1582.

19 Clarkson MR, Giblin L, Brown A, Little D, Donohoe J: Reversal of pulmonary hypertension after ligation of a brachiocephalic arteriovenous fistula. Am J Kidney Dis 2002; 40:E8.

20 Yigla M, Abassi Z, Reisner SA, Nakhoul F: Pulmonary hypertension in hemodialysis patients: an unrecognized threat. Semin Dial 2006;19:353-357.

21 Bos WJ, Zietse R, Wesseling KH, Westerhof $\mathrm{N}$ : Effects of arteriovenous fistulas on cardiac oxygen supply and demand. Kidney Int 1999;55:2049-2053.

22 Crowley S, Butterly D, Peter R, Schwab S: Coronary steal from a left internal mammary artery coronary artery bypass graft by a left upper extremity arteriovenous hemodialysis fistula. Am J Kidney Dis 2002;40:852855.
23 Schneditz D, Kaufman AM, Polaschegg HD, Levin NW, Daugirdas JT: Cardiopulmonary recirculation during hemodialysis. Kidney Int 1992;42:1450-1456.

24 van der Mark WA, Boer P, Cramer MJ, Blankestijn PJ: Decreased access resistance in haemodialysis patients with upper arm arteriovenous fistulae. Nephrol Dial Transplant 2008;23:2105-2106.

25 MacRae JM, Levin A, Belenkie I: The cardiovascular effects of arteriovenous fistulas in chronic kidney disease: a cause for concern? Semin Dial 2006;19:349-352.

26 Abbott KC, Trespalacios FC, Agodoa LY: Arteriovenous fistula use and heart disease in long-term elderly hemodialysis patients: analysis of United States Renal Data System Dialysis Morbidity and Mortality Wave II. J Nephrol 2003;16:822-830.

27 Combe C, Pisoni RL, Port FK, Young EW, Canaud B, Mapes DL, Held PJ: Dialysis Outcomes and Practice Patterns Study: data on the use of central venous catheters in chronic hemodialysis. Nephrologie 2001;22:379384.

28 Unger P, Wissing KM, De Pauw L, Neubauer J, van de Borne P: Reduction of left ventricular diameter and mass after surgical arteriovenous fistula closure in renal transplant recipients. Transplantation 2002;74:73-79.

29 van Duijnhoven, Cheriex ECM, Tordoir JHM, Kooman JP, van Hoof J: Effect of closure of the arteriovenous fistula on left ventricular dimensions in renal transplant patients. Nephrol Dial Transplant 2001;16: 368-372.

30 Movilli, et al: Long-term effects of AVF closure on echocardiographic functional and structural findings in HD patients: a prospective study. Am J Kidney Dis 2010;55: 682-689.

31 Amerling R: Fistula first: is it time to curb our enthusiasm? Neph News Issues 2006;20: 38-39.

32 Wolford HY, Hsu J, Rhodes JM, Shortell CK, Davies MG, Bakhru A, Illig KA: Outcome after autogenous brachial-basilic upper arm transpositions in the post-National Kidney Foundation Dialysis Outcomes Quality Initiative era. J Vasc Surg 2005;42:951-956. 